\title{
Analytical exact solutions for unsteady collisionless plume flows in a vacuum is
}

\author{
C. Cai ${ }^{\mathrm{a}, *}$, Q. Sun ${ }^{\mathrm{b}}$, A. Vanderwyst ${ }^{\mathrm{c}}$ \\ ${ }^{a}$ Department of Mechanical E' Aerospace Engineering, Jett Hall 515, 1040 S. Horseshoe Dr., MSC 3450, New Mexico State University, Las \\ Cruces, New Mexico 88003, United States \\ b State Key Laboratory of High Temperature Gas Dynamics, No. 15 Beisihuanxi Road, Institute of Mechanics, Chinese Academy of Sciences, \\ Beijing 100190, China \\ ' Sr. AIAA Member in Tucson, AZ 85716, United States
}

\section{A R T I C L E I N F O}

\section{Article history:}

Received 20 February 2013

Received in revised form

15 June 2013

Accepted 18 June 2013

Available online 26 June 2013

Keywords:

Rarefied jet flows

Jets and bursts

Collisionless

Discharge in vacuum

\begin{abstract}
A B S T R A C T
This paper presents a set of gaskinetic solutions to the problem of unsteady collisionless round plume development: startup and development to a steady flow; steady flow; and shutting down from a steady flow. This can find applications in studying similar transient plume flows from space propulsion devices. Different from many past studies, here we consider the general situation that the average exit gas speed can be larger than zero, and detailed geometry of the nozzle exit radius is included. A fundamental space-velocitytime relation is adopted in this study and it plays a crucial role to obtain the complete flowfield properties of density, velocity, pressure and temperature. This study reveals that there are some internal complementary relations on density and momentum among these three processes. The results involve complex integrations involving factors of time, geometry, and specific speed ratio. Several numerical simulations with the direct simulation Monte Carlo method validate these analytical exact results.
\end{abstract}

Published by Elsevier Ltd. on behalf of IAA

\section{Introduction}

High speed collisionless flows from small holes are a fundamental fluid dynamic problem with many applications, including electronic beams [1,2], materials processing inside vacuum chambers [3], spacecraft design, gaseous sprays, electric propulsion [4], micro-propulsion [5], and metrology gas flows. Many investigators have presented analytical expressions for the gas mass flow rate through an orifice expanding into a vacuum. Just to name a few, one of the earliest to report the efflux of gases through circular apertures was by Liepmann [6]; Narasimha [7] further obtained the exact solutions of density

\footnotetext{
This is a collaborative research result.

* Corresponding author. Tel.: +1 575646 7704; fax: +1 5756466111 .

E-mail address: ccai@nmsu.edu (C. Cai).
}

and velocity distributions for free molecular flows between two chambers with different background pressures, and steady state gas out of a one-dimensional exit [8]; Brook [9] studied the gas leakage effect from a missealed spacecraft hatch by using free molecular flow from an annulus, he assumed that the average exit velocity was zero. Woronowicz presented [10] some concise results for one-dimensional unsteady free jet expansion into a vacuum. There are many existing experimental measurements and numerical simulations of plasma plumes, e.g. for materials processing. The widely used cosine law or the Boynton/Simons plume model $[11,12]$ provides us an approximate farfield density distribution which takes a form of a cosine function. However, in the literature there are very rare analytical formulas that consider the detailed exit geometries, high exit speed, and time. Most of the previous work concentrates on free molecular flows out of a highly simplified geometry and with a zero 
average exit velocity, $U_{0}=0$. In addition, many of these results only offered farfield flow predictions and neglected the influence of exit geometries. Dettleef [13] provided a detailed review for plume and plume impingement in space engineering.

There are some recent interesting developments on analytical, high speed steady free molecular flows out of a round exit [14]. For many applications, the jet/plume flows are highly rarefied and the average velocities at the exit are high, examples include electric propulsion plume flows, e.g. jet flows from an ion thruster or a Hall thruster, a Pulsed Plasma Thruster (PPT), and materials processing with thin file depositions inside a vacuum chamber. A recent study [15] indicates that for high speed jet/plume flows, we can use the high Knudsen(Kn) number flow solutions [14] to approximate them, even though these jets or plumes are near continuum. This is due to the general relation among $\mathrm{Kn}, \mathrm{Ma}$ and $\mathrm{Re}$ numbers

$K n \sim M a / R e$,

or physically, if the exit speed is high, molecules flow downstream-wise so quickly that they do not have time to collide and diffuse vertically. Indeed, it was illustrated that even if the Knudsen number changes four or more orders, from 100 to 0.01 , the jet flowfield does not change much [15]. This conclusion is obtained from the steady collisionless flow situation, however, we do not see a reason that it would not be right for an unsteady jet or plume flow.

This paper is a continued effort to further investigate the full set of exact solutions to a high speed, round collisionless jet flow, and the solutions are valid for the whole flowfield. Because the results are very complex, we also included several numerical validations with the direct simulation Monte Carlo (DSMC) method [16]. The motivation for this work is very simple: there are many unsteady jet flows cannot be well approximated with steady flow solutions; every gaseous jet/plume flow must have a startup process and a shut down process, even though it is not necessary to have a steady flow state. One good example is Pulsed Plasma Thruster (PPT) plume flows. The results in this papers will supersede the past steady jet flow solutions [14], by further including the factor of time; therefore the results are more realistic and general. We adopted a relation among velocity, location and time, allowing us to perform a mathematical variable transformation from velocity phase $(u, v, w)$ to the round nozzle exit geometry.

Section 2 presents the problem to be studied, and a brief summary of some recent steady flow results; Section 3 presents the solutions to the problems of collisionless jet startup and shutting down; Section 4 compares the analytical and DSMC simulation results; and Section 5 summarizes this study.

\section{Steady round jet flowfield solutions with an average exit speed $U_{0}>0$}

The flows considered in this study are transient free molecular gases leaving a round/annular exit into a vacuum, the exit speed is larger than zero. They include three stages: starting up and developing towards a steady flow; steady flow; and shutting down from a steady state. The foundations and solutions to the jet startup and shutting down process problems are the steady state flow problem. As such, first we perform a brief review on the steady jet flow problem and solutions.

Fig. 1 illustrates molecules flow out of a circular exit of a radius of $R_{n}$, with an average exit velocity $U_{0}$. Round jet flows are special because they are most commonly used, and the easiest duct shape to manufacture. An annular exit is a more general scenario than a round jet but an annular one can illustrate the results better with more variations in flowfield patterns. The methods and results in this study are applicable to both round and annular exits. The coordinate system is common in the field, with the jet moving along $+X$-axis, being normal to the exit plane and $(0,0,0)$ the center point of the exit. To simplify the problem, we assume that the gas at the exit is in equilibrium with a Maxwellian velocity distribution function (VDF) which is characterized with a uniform static temperature $T_{0}$ and a uniform number density $n_{0}$, and we concentrate on the results for a flowfield point $P(X, 0, Z)$ due to the symmetry $[14,15]$.

Fig. 2 illustrates the velocity spaces for a point $P(X, 0, Z)$ in front of the exit. This figure actually can display the three jet flow problems. For a steady flow state, the velocity domain $\Omega$ must be an infinitely long, full oblique cone extending to the right, and it is symmetric about the

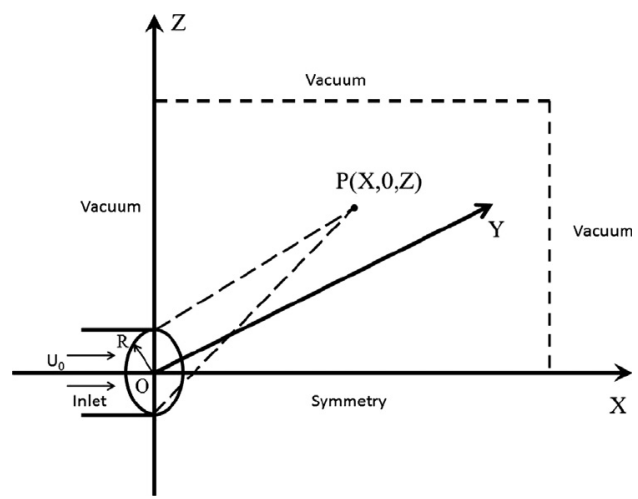

Fig. 1. Illustrations for the problems of round jet flow and the computation domain.

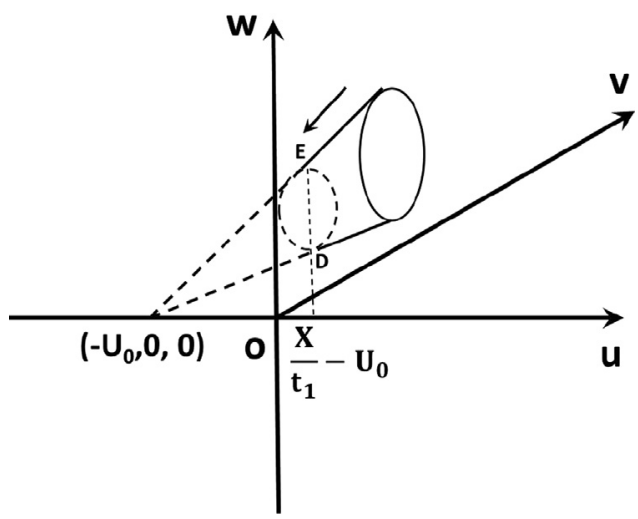

Fig. 2. Illustration for the velocity phase domain, round jet starting up scenario. 
$v=0$ plane. The vertex point of the oblique-cone alike velocity shape in the figure is shifted to the left by $U_{0}[14]$. From a point on the exit, only particles with unique velocity components can arrive at a specific point $P(X, 0, Z)$ in front of the exit. The related VDF to be integrated is [14]

$f(u, v, w)= \begin{cases}n_{0}\left(\frac{\beta_{0}}{\pi}\right)^{3 / 2} e^{-\beta_{0}\left(u^{2}+v^{2}+w^{2}\right)}, & u, v, w \in \Omega_{p} ; \\ 0, & u, v, w \notin \Omega_{p} .\end{cases}$

With a known velocity distribution $f(u, v, w)$ at a point $P(X, 0, Z)$, we can obtain the average flowfield properties of number density, velocity, and pressure. The velocity components of those particles which start from a point $(0, y, z)$ at the circular nozzle and arrive at point $P(X, 0, Z)$ must satisfy the following constraint condition $[14,15]$ :

$\frac{X-0}{u+U_{0}}=\frac{0-y}{v}=\frac{Z-z}{w}$,

here, $y=r \cos \theta, z=r \sin \theta ; r, \theta$ are the polar coordinate variables for the exit plane. Bearing in mind that the velocity phase for point $P(X, 0, Z)$ has an oblique-cone shape with an infinite length, it is desirable to utilize a change of variables to transform the infinite integration domain of velocity to a finite one of the exit. From the above constraint condition, we have

$v=\frac{-r \cos \theta}{X}\left(u+U_{0}\right) ; \quad w=\frac{Z-r \sin \theta}{X}\left(u+U_{0}\right)$,

and

$d u d v d w=\frac{\left(u+U_{0}\right)^{2}}{X^{2}} r d u d r d \theta$,

then the final results for the flowfield density, velocity and temperatures are $[14,15,17]$

$$
\begin{aligned}
& n_{s}(X, 0, Z) / n_{0}=\frac{e^{-S_{0}^{2}}}{\sqrt{\pi^{3}} X^{2}} \int_{-\pi / 2}^{\pi / 2} d \theta \int_{0}^{R_{n}} r K d r \\
& U_{s}(X, 0, Z) \sqrt{\beta_{0}}=\frac{e^{-S_{0}^{2}}}{\sqrt{\pi^{3}} X^{2}} \frac{n_{0}}{n_{s}} \int_{-\pi / 2}^{\pi / 2} d \theta \int_{0}^{R_{n}} r M d r \\
& W_{s}(X, 0, Z) \sqrt{\beta_{0}}=\frac{e^{-S_{0}^{2}}}{\sqrt{\pi^{3}} X^{3}} \frac{n_{0}}{n_{s}} \int_{-\pi / 2}^{\pi / 2} d \theta \int_{0}^{R_{n}}(Z-r \sin \theta) r M d r
\end{aligned}
$$

$$
\begin{aligned}
T_{s}(X, 0, Z) / T_{0}= & -\frac{U_{s}^{2}+W_{s}^{2}}{3 R T_{0}} \\
& +\frac{4}{3} \frac{e^{-S_{0}^{2}}}{\sqrt{\pi^{3}} X^{2}} \frac{n_{0}}{n_{s}} \int_{-\pi / 2}^{\pi / 2} d \theta \int_{0}^{R_{n}} N r d r,
\end{aligned}
$$

with the following integrand factors:

$$
\begin{aligned}
Q & =\frac{X^{2}}{X^{2}+Z^{2}+r^{2}-2 Z r \sin \theta} \\
& =\cos ^{2} \psi\left[\sum_{n=0}^{\infty} P_{n}(\sin \psi \sin \theta)\left(\frac{r}{\sqrt{X^{2}+Z^{2}}}\right)^{n}\right]^{2}, \\
K & =Q^{2} S_{0}+\left(\frac{1}{2}+Q S_{0}^{2}\right) \sqrt{\pi Q^{3}}\left[1+\operatorname{erf}\left(S_{0} \sqrt{Q}\right)\right] e^{S_{0}^{2} Q},
\end{aligned}
$$

$$
M=Q^{2}\left[Q S_{0}^{2}+1+S_{0}\left(\frac{3}{2}+Q S_{0}^{2}\right) \sqrt{\pi Q}\left[1+\operatorname{erf}\left(S_{0} \sqrt{Q}\right)\right] e^{S_{0}^{2} Q}\right]
$$

$$
\begin{aligned}
N= & S_{0} Q^{2}\left[\frac{5}{4}+\frac{Q S_{0}^{2}}{2}\right]+\frac{1}{2} \sqrt{Q^{3} \pi}\left(\frac{3}{4}+3 Q S_{0}^{2}+Q^{2} S_{0}^{4}\right) \\
& {\left[1+\operatorname{erf}\left(S_{0} \sqrt{Q}\right)\right] e^{S_{0}^{2} Q}, }
\end{aligned}
$$

where "s" represents steady state properties. $P_{n}() s$ are the Legendre polynomials, $\psi=\arctan (Z / X), \quad S_{0}=U_{0} / \sqrt{2 R T_{0}}$. These formulae illustrate that the free plume flowfield solutions contain geometry factors of $R_{n}, X, Z$, and the velocity factor $S_{0}$. As can be seen, the problem of a three-dimensional rarefied free plume expanding from a circular exit has compact solutions. The flowfield solutions are complete with $\rho, U, W, P$; the integration is fast because the finite integration domain is two-dimensional, not three-dimensional; also the integration accuracy is enhanced because the integration domain is finite with geometry factors rather than an open domain with an infinite length.

It is worthwhile to compare these round jet results with some past popular plume models. Eqs. (5)-(8) are exact solutions to collisionless flows, but they are extendable to near-continuum high speed flows into a vacuum, for the whole flowfield. While the past widely used cosine law/Boynton/Simons plume model is a point source approximation for a far-field plume, and for density only

$\frac{\rho(r, \theta)}{\rho_{s}} \sim \frac{1}{r^{n}} \cos ^{\kappa}\left(\frac{\pi}{2} \frac{\theta}{\theta_{\max }}\right)$,

where $r, \theta$ are the polar coordinate variables formed by a field point and the exit origin point; $\kappa$ is the so-called beam factor, different people used $\kappa=2,1 /(\gamma-1)$, or $2 /(\gamma-1)$ for different problems.

One recent paper provided some detailed comparisons among exact analytical solutions, Eqs. (5)-(8), and several less rarefied plume DSMC simulations [15]. In addition to that, here we present some brief comparisons on planar jet scenario.

Fig. 3 compares some plume flow centerline density profiles, predicted by exact analytical solutions

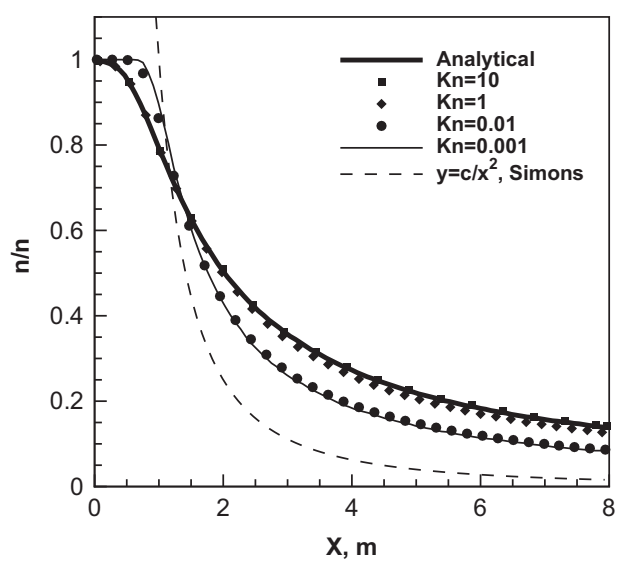

Fig. 3. Planar jet centerline density: analytical (collisionless), DSMC simulations (collisionless to near continuum), and the Boynton/Simons' model (continuum). 


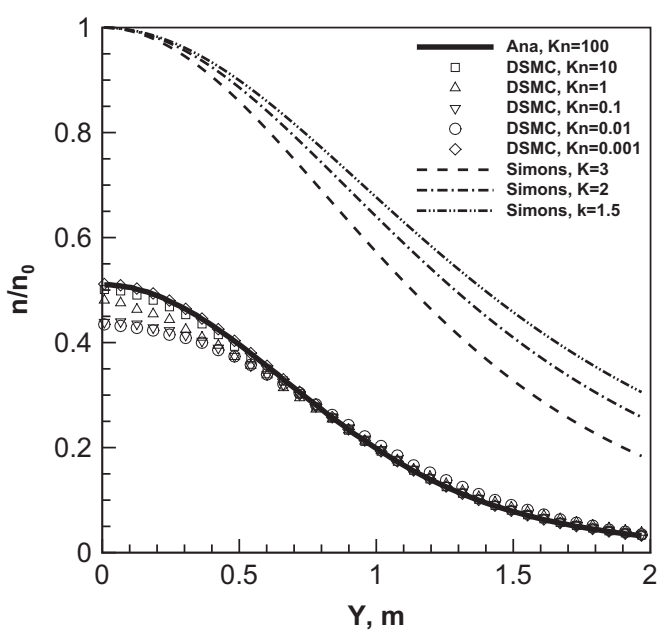

Fig. 4. Planar jet density along $X=2 \mathrm{~m}$ : analytical (collisionless), DSMC simulations (collisionless to near continuum), and Boynton/Simons model (continuum) with different $\kappa$.

(collisionless flows), less rarefied plumes (even including continuum flows) simulated with the DSMC method, and a Boynton/Simons alike formula(continuum). Along the jet centerline, the cosine law/Boynton/Simons model degenerates to $\rho(r, 0) \sim 1 / r^{n}$. From this figure, we can observe that: (i) the Boynton/Simons model diverges at the nozzle exit due to the $1 / r^{n}$ factor; (ii) the curvature of the Boynton/ Simons model is opposite to all other curves; (iii) the analytical solution and the DSMC simulation results share the same starting value and follow similar trends. We can fairly conclude that the analytical solutions can represent the real steady, high speed, rarefied, round jet flows into a vacuum better.

Fig. 4 shows comparisons of density profiles from a planar jet, along a vertical lines of $X=2 \mathrm{~m}$. It includes some DSMC simulations, the analytical collisionless flow solutions, and a Boynton/Simons alike plume model results. As can be seen, the analytical results can be used to approximate near continuum flows: even the $K n$ numbers are different, the normalized off-centerline density profiles degenerate and merge into one curve. By comparison, the Boynton/Simons alike model has different profiles due to different beam factors. Due to the real $K n$ number effects, the numerical simulation results have different centerline starting values, but it is possible that we correct these values by using a modification factor which is related with the $K n$ number.

These two figures illustrate that the cosine law plume model is not ideal to simulate the problem of high speed, rarefied jet into a vacuum, but the exact collisionless flow solutions can approximate the jet flows much better.

\section{Unsteady collisionless round jet startup and shutdown}

This section discusses the other two problems of jet startup and jet shutting down, first we revert to Fig. 2 to discuss a jet starting up. Particles starting from a point $(0, y, z)$ at the exit arrive at point $P(X, 0, Z)$ at time $t$ if their velocity components satisfy the following extra relations:
$X /\left(u+U_{0}\right)=\tau,(0-y) / v=\tau$ and $(Z-z) / w=\tau$, with $\tau<t$. They actually split the large oblique cone alike velocity phase in Fig. 2 into two parts. The left side of the cone with dashed lines is for those molecules that have not arrived at point $P(X, 0, Z)$ due to their slow speeds. As such, the left side of plane ED can be used to represent the shutdown portion of particles, when the ED plane moving to the left. The right portion of the oblique cone, represents those fast molecules that have already reached $P(X, 0, Z)$ and contribute to the flowfield properties. By merging these three constraining relations, we obtain the following velocityspace-time relation:

$\frac{X}{u+U_{0}}=\frac{Y-y}{v}=\frac{Z-z}{w}=\tau$.

This relation restricts the integration domain to the left portion of the oblique cone-alike velocity phase, $u \in\left(-U_{0}, X / t-U_{0}\right)$. It can be proven, similar to the steady flow case, the properties for an unsteady shutting down process, at point $P(X, 0, Z)$ and time $t$ is

$$
\begin{aligned}
& n_{e}(X, 0, Z ; t) \\
& \quad=\frac{2 n_{0} e^{-S_{0}^{2}}}{\sqrt{\pi}^{3} X^{2}} \int_{0}^{\sqrt{\beta_{0}} X / t} \int_{0}^{R_{n}} r d r \int_{-\pi / 2}^{\pi / 2} d \theta s^{2} e^{-s^{2} / Q+2 s S_{0}} d s,
\end{aligned}
$$

$$
\begin{aligned}
& \left(n_{e} U_{e}\right)(X, 0, Z ; t) \sqrt{\beta_{0}} \\
& \quad=\frac{2 n_{0} e^{-S_{0}^{2}}}{\sqrt{\pi}^{3} X^{2}} \int_{0}^{\sqrt{\beta_{0}} X / t} \int_{0}^{R_{n}} r d r \int_{-\pi / 2}^{\pi / 2} d \theta s^{3} e^{-s^{2} / Q+2 s S_{0}} d s,
\end{aligned}
$$

$$
\begin{aligned}
& \left(n_{e} W_{e}\right)(X, 0, Z ; t) \sqrt{\beta_{0}} \\
& \quad=\frac{2 n_{0} e^{-S_{0}^{2}}}{\sqrt{\pi}^{3} X^{3}} \int_{0}^{\sqrt{\beta_{0} X / t}} \int_{0}^{R_{n}} r d r \int_{-\pi / 2}^{\pi / 2} d \theta(Z-r \sin \theta) s^{3} e^{-s^{2} / Q+2 s S_{0}} d s,
\end{aligned}
$$

$$
\begin{aligned}
& \frac{P_{e}}{p_{0}}(X, 0, Z ; t)=-\frac{U_{e}^{2}+W_{e}^{2}}{3 R T_{0}} \frac{n_{e}}{n_{0}} \\
& \quad+\frac{4}{3} \frac{e^{-S_{0}^{2}}}{\sqrt{\pi} X^{2}} \int_{0}^{\sqrt{\beta_{0}} X / t} \int_{0}^{R_{n}} r d r \int_{-\pi / 2}^{\pi / 2} d \theta \frac{s^{4}}{Q} e^{-s^{2} / Q+2 s S_{0}} d s .
\end{aligned}
$$

Here “ $e$ " represents a shutting down process property over a domain, it contains factors of exit geometry, time and speed ratio. The above results are convenient for computer evaluations. To reduce the computation cost, we can further integrate those "s" factors out and obtain several finite integrations. However, the results are very complex. By using those complex integration formulas in the appendix, we have

$$
\begin{aligned}
& n_{e}(X, 0, Z ; t)=\frac{2 n_{0} e^{-S_{0}^{2}}}{\sqrt{\pi}^{3} X^{2}} \int_{0}^{R_{n}} r d r \int_{-\pi / 2}^{\pi / 2} d \theta\left[\frac{Q^{2} S_{0}}{2}\right. \\
& -\left(\frac{Q \sqrt{\beta_{0}} X}{2 t}+\frac{Q^{2} S_{0}}{2}\right) e^{-\beta_{0} X^{2} / t^{2} Q+2 \sqrt{\beta_{0}} X / t S_{0}} \\
& \left.+\frac{\sqrt{\pi Q^{3}}}{4}\left(1+2 S_{0}^{2} Q\right) e^{S_{0}^{2} Q}\left[\operatorname{erf}\left(\frac{\sqrt{\beta_{0}} X}{t \sqrt{Q}}-\sqrt{Q} S_{0}\right)+\operatorname{erf}\left(\sqrt{Q} S_{0}\right)\right]\right],
\end{aligned}
$$




$$
\begin{aligned}
& \left(n_{e} U_{e}\right)(X, 0, Z ; t)=\frac{2 n_{0} e^{-S_{0}^{2}}}{\sqrt{\beta_{0}} \sqrt{\pi}^{3} X^{2}} \int_{0}^{R_{n}} r d r \int_{-\pi / 2}^{\pi / 2} \\
& \times d \theta\left[-\frac{Q}{2}\left(\frac{\beta_{0} X^{2}}{t^{2}}+Q+Q S_{0} \frac{\sqrt{\beta_{0}} X}{t}+Q^{2} S_{0}^{2}\right)\right. \\
& \times e^{-\beta_{0} X^{2} / t^{2} Q+2 \sqrt{\beta_{0}} X / t S_{0}}+\left[3 Q S_{0}+2 Q^{2} S_{0}^{3}\right] \frac{\sqrt{\pi Q^{3}}}{4} e^{S_{0}^{2} Q} \\
& \left.\quad \times\left[\operatorname{erf}\left(\frac{\sqrt{\beta_{0}} X}{t \sqrt{Q}}-\sqrt{Q} S_{0}\right)+\operatorname{erf}\left(\sqrt{Q} S_{0}\right)\right]+\frac{Q^{2}}{2}\left(1+Q S_{0}^{2}\right)\right],
\end{aligned}
$$

$$
\begin{aligned}
& \left(n_{e} W_{e}\right)(X, 0, Z ; t)=\frac{2 n_{0} e^{-S_{0}^{2}}}{\sqrt{\beta_{0}} \sqrt{\pi} X^{3}} \int_{0}^{R_{n}}(Z-r \sin \theta) r d r \int_{-\pi / 2}^{\pi / 2} \\
& \times d \theta\left[\frac{Q^{2}}{2}\left(1+Q S_{0}^{2}\right)-\frac{Q}{2}\left(\frac{\beta_{0} X^{2}}{t^{2}}+Q+Q S_{0} \frac{\sqrt{\beta_{0}} X}{t}+Q^{2} S_{0}^{2}\right)\right. \\
& \times e^{-\beta_{0} X^{2} / t^{2} Q+2 \sqrt{\beta_{0}} X / t S_{0}}+\left[3 Q S_{0}+2 Q^{2} S_{0}^{3}\right] \\
& \left.\quad \times \frac{\sqrt{\pi Q^{3}}}{4} e^{S_{0}^{2} Q}\left[\operatorname{erf}\left(\frac{\sqrt{\beta_{0}} X}{t \sqrt{Q}}-\sqrt{Q} S_{0}\right)+\operatorname{erf}\left(\sqrt{Q} S_{0}\right)\right]\right],
\end{aligned}
$$$$
\frac{P_{e}(X, 0, Z ; t)}{p_{0}}=-\frac{U_{e}^{2}+W_{e}^{2}}{3 R T_{0}} \frac{n_{e}}{n_{0}}-\frac{4}{3} \frac{e^{-S_{0}^{2}}}{\sqrt{\pi}^{3} X^{2}} \int_{0}^{R_{n}} r d r \int_{-\pi / 2}^{\pi / 2}
$$$$
\times d \theta\left[\frac{Q}{2}\left(\frac{\sqrt{\beta_{0}} X}{t}\right)^{3}+\frac{3}{2} Q \frac{\sqrt{\beta_{0}} X}{t}+\frac{5}{2} Q^{2} S_{0}+Q S_{0} \frac{\beta_{0} X^{2}}{t^{2}}\right.
$$$$
\left.+Q^{2} S_{0}^{2} \frac{\sqrt{\beta_{0}} X}{t}+S_{0}^{3} Q^{3}\right) e^{-\beta_{0} X^{2} / t^{2} Q+2 \sqrt{\beta_{0}} X / t S_{0}}
$$$$
-\frac{\sqrt{\pi Q^{3}}}{4} e^{S_{0}^{2} Q}\left(\frac{3}{2} Q+6 Q^{2} S_{0}^{2}+2 Q^{3} S_{0}^{4}\right)\left[\operatorname{erf}\left(\frac{\sqrt{\beta_{0}} X}{t \sqrt{Q}}-\sqrt{Q} S_{0}\right)\right.
$$$$
\left.\left.+\operatorname{erf}\left(\sqrt{Q} S_{0}\right)\right]-\frac{Q^{3}}{4}\left(2 Q S_{0}^{3}+5 S_{0}\right)\right] \text {. }
$$

These new formulas reduce the integration domain, from an open three-dimensional $(u, v, w)$ (extending to infinity), to one finite range evaluation of $r, \theta$ for the exit. Hence both the computation accuracy and the speed are greatly enhanced. It is also observable that when time is infinitely long, all properties approach to zero. This means the jet completely stops. This state will never reach because there will always be extremely slow particles from the exit to arrive this flowfield point.

From Fig. 2 actually we can observe some internal relations among the processes of jet startup and shutting down: their unsteady phase velocity domains are the two parts of a full cone alike domain for a steady jet, as such, they are complementary. From this relation and the gaskinetic definitions of density and momentums, we can immediately obtain that their summation shall be the steady state results. However, we shall not use this relation for pressure computations.

The startup process can be computed similarly and the results are

$n_{b}(X, 0, Z ; t)=n_{s}-\frac{2 n_{0} e^{-S_{0}^{2}}}{\sqrt{\pi}^{3} X^{2}} \int_{0}^{\sqrt{\beta_{0}} X / t} \int_{0}^{R_{n}} r d r$

$$
\begin{aligned}
& \int_{-\pi / 2}^{\pi / 2} d \theta s^{2} e^{-s^{2} / Q+2 s S_{0}} d s, \\
& \left(n_{b} U_{b}\right)(X, 0, Z ; t)=n_{s} U_{s}-\frac{2 n_{0} e^{-S_{0}^{2}}}{\sqrt{\beta_{0}} \sqrt{\pi^{3}} X^{2}} \int_{0}^{\sqrt{\beta_{0}} X / t} \int_{0}^{R_{n}} r d r \\
& \times \int_{-\pi / 2}^{\pi / 2} d \theta s^{3} e^{-s^{2} / Q+2 s S_{0}} d s, \\
& \left(n_{b} W_{b}\right)(X, 0, Z ; t)=n_{s} W_{s}-\frac{2 n_{0} e^{-S_{0}^{2}}}{\sqrt{\beta_{0}} \sqrt{\pi} X^{3}} \int_{0}^{\sqrt{\beta_{0}} X / t} \\
& \quad \times \int_{0}^{R_{n}} r d r \int_{-\pi / 2}^{\pi / 2} d \theta(Z-r \sin \theta) s^{3} e^{-s^{2} / Q+2 s S_{0}} d s, \\
& \frac{P_{b}(X, 0, Z ; t)}{p_{0}}=-\frac{U_{b}^{2}+W_{b}^{2}}{3 R T_{0}} \frac{n_{b}}{n_{0}}+\frac{P_{s}-P_{e}(X, 0, Z ; t)}{p_{0}} \\
& +\frac{U_{s}^{2}+W_{s}^{2}}{3 R T_{0}} \frac{n_{s}}{n_{0}}-\frac{U_{e}^{2}+W_{e}^{2}}{3 R T_{0}} \frac{n_{e}}{n_{0}},
\end{aligned}
$$

where " $b$ " represents a starting up process property. These unsteady results are more general than the steady solutions, Eqs. (5)-(8), due to the unsteady term. Meanwhile, these results are applicable for the entire flow field in space.

The above results of jet starting up are different from the past results by Bender [18]. First, our results are based on the past solutions for high speed, high $K n$ number, steady, round jet; second the new results of start up are for the whole flowfield while Bender results are limited to centerline; third, the results in this paper can fully integrate out the time factor but reserve the $\theta$ factor; while Bender's results keep the time factor as an integral variable but integrate the $\theta$ out, which only holds along the centerline.

Before we proceed to the next section for validations, we compare several formulas for the density distribution along the jet centerline, to demonstrate the difference among this paper and the previous ones in the literature.

The first one is the traditional, zero exit average velocity $S_{0}=0$, collisionless, steady, circular hole problem with $R_{n}=0$, in the literature (e.g. [16]):

$\frac{n_{1}(X)}{n_{0}}=\frac{1}{2}\left(1-\frac{X}{\sqrt{X^{2}+R_{n}^{2}}}\right)$,

the second one is the traditional one-dimensional, zero average exit velocity $S_{0}=0$, collisionless, unsteady, circular hole with $R_{n}=0$, shutting down problem, in the literature [16]:

$\frac{n_{2}(X, t)}{n_{0}}=\frac{1}{2} \operatorname{erfc}\left(\frac{\sqrt{\beta_{0}} X}{t}\right)$,

the third one is the non-zero average exit speed, $S_{0}>0$, collisionless, steady, from a finite size circular exit with $R_{n}>0$, obtained recently [14]:

$\frac{n_{3}(X)}{n_{0}}=\frac{1}{2}+\frac{1}{2} \operatorname{erf}\left(S_{0}\right)-\frac{X}{2 \sqrt{X^{2}+R_{n}^{2}}} e^{-S_{0}^{2} /\left(X / R_{n}\right)^{2}+1}$ 


$$
\times\left[1+\operatorname{erf}\left(\frac{S_{0} X}{\sqrt{X^{2}+R_{n}^{2}}}\right)\right],
$$

the fourth one is the non-zero average exit speed, $S_{0}>0$, collisionless, unsteady, circular exit with $R_{n}>0$, jet shutting down problem, from this paper

$$
\begin{aligned}
& \frac{n_{4}(X, t)}{n_{0}}=\frac{e^{-S_{0}^{2}}}{2 \sqrt{\pi}} \frac{X^{2}}{X^{2}+R_{n}^{2}}-\frac{e^{-S_{0}^{2}}}{\sqrt{\pi} X^{2}} \\
& \quad \times \int_{0}^{R_{n}} r d r\left[\left(\frac{Q_{1} \sqrt{\beta_{0}} X}{t}+Q_{1}^{2} S_{0}\right) e^{-\beta_{0} X^{2} / t^{2} Q_{1}+2 \sqrt{\beta_{0}} X / t S_{0}}\right. \\
& \left.\quad+\frac{\sqrt{\pi Q_{1}^{3}}}{2}\left(1+2 S_{0}^{2} Q_{1}\right) e^{S_{0}^{2} Q_{1}}\left[\operatorname{erf}\left(\frac{\sqrt{\beta_{0}} X}{t \sqrt{Q_{1}}}-\sqrt{Q_{1}} S_{0}\right)+\operatorname{erf}\left(\sqrt{Q_{1}} S_{0}\right)\right]\right],
\end{aligned}
$$

here we define $Q_{1}=X^{2} /\left(X^{2}+r^{2}\right)$.

\section{Simulations and discussion}

Although the above complex results involve several integral terms that cannot be explicitly evaluated, to evaluate these concrete and explicit formulas is relatively faster and convenient than numerical simulations. Because the flows in this study are highly rarefied, the DSMC method is one of the most feasible simulation methods. Experimental results are not ideal because collisions are inherently included. In this study, we used a specific DSMC package named GRASP-P [19], which was developed with ObjectedOriented-Programming style, to perform the simulations. The collision function in GRASP is turned off. As the results, the value of the molecular exit number density is irrelevant and true free molecular flows are created. Here we emphasize that this paper is about analytical studies or development on new unsteady jet flows into a vacuum, and numerical simulations are only used to validate the results -readers shall not consider this paper on computational fluid dynamics.

We chose round and/or annular exit shapes to perform the simulations. The simulation domain is illustrated in Fig. 1, and the boundary conditions are very simple: the bottom side is an axis symmetric line; the left bottom corner is an inlet to the flowfield with zero exit thickness, i.e., the exit nozzle has no length, representing a round or an annular exit; while the rest portion of the left side, the top and the right sides are set to vacuum conditions. At the inlet, we assume that the gas is at equilibrium state and it is approximated with a Maxwellian distribution function which is characterized by number density, temperature and average exit velocity.

For the DSMC simulations, the characteristic exit speed ratio is set to $S_{0}=2.0$, at exit the gas temperature is set to $T_{0}=300 \mathrm{~K}$. The circular radius or the annular width is set to $0.5 \mathrm{~m}$. These analytical results we obtained and listed in the previous sections are exact from a mathematical point of view, but they highly depend on the incoming boundary conditions.

For a steady jet flow, to reduce the statistical scatters in the DSMC simulation results, we can use many sampling steps. For the unsteady flows of jet starting up and shutting down, we cannot use too many time steps, neither a large time step, during the sampling process; instead we used a relatively large number of particles, fewer sampling steps, and a shorter marching time increment for sampling. A large sampling size requires a larger computing machine, or we shall use an improved DSMC method; both are active research topics right now. Bearing these challenges in mind, these new analytical formulas show their outstanding features over DSMC simulations: e.g. for our first test case with $t / T_{*}=0.25$, it only took several seconds to evaluate, but with the same computer, it took more than $10 \mathrm{~min}$ to evaluate with the DSMC methods. Of course, the later depends on different number of particles and time steps. The present DSMC results are time-averaged results over a small time span, and the time span will be given wherever needed.

Fig. 5 shows the steady state number density for a round jet flow with $K n=100$. The results include DSMC simulation data, analytical formula (Eq. (5)), and the cosine law plume model. In general, the DSMC and analytical results have essentially identical agreement, but the cosine law/Boynton/Simons plume model has problems at the jet exit plane. The plume expands quickly into a vacuum and the exit lip is a singularity point with very large gradients.

Fig. 6 shows the steady state $U$-velocity component. Without any inter-molecule collisions, all particles from the jet exit move forward, and no negative $U$-velocity component observed. This picture also shows symmetric patterns at the centerline axis, with $\partial(U) / \partial n=0$. The exit lip is still a s ingularity point because the velocity components change from very large values to almost zero just above the lip because of no particles moving upward. This picture also illustrates that a large portion of diffusion is due to the differences among particle velocities, rather than inter-molecular collisions. Inside the plume core, the average molecule speeds approach to an asymptote.

Fig. 7 illustrates the $V$-velocity component for a round jet into a vacuum. Results from the analytical expression of Eq. (7) are on the top while the DSMC simulation results are at bottom. Along the axis centerline, the $V$-velocity component must be zero due to the symmetric boundary condition. Along the vertical line above the exit, the

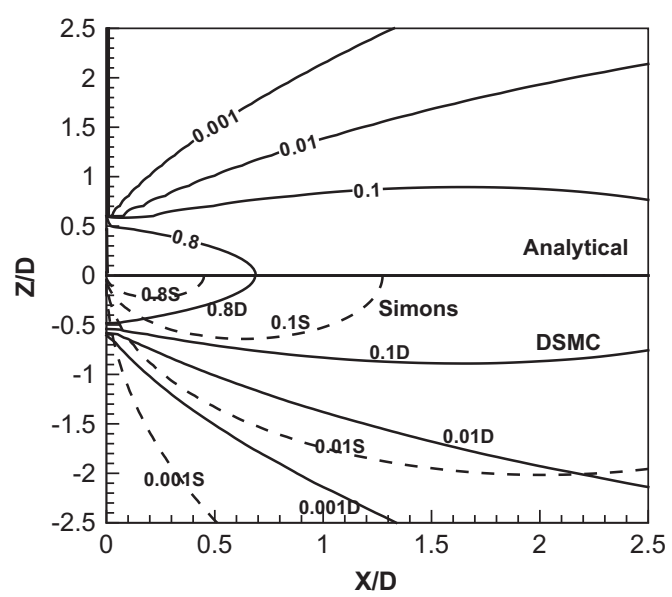

Fig. 5. Steady round jet: normalized number density, $S_{0}=2.0$. Solid: DSMC (bottom), analytical (top); dashed: Boynton/Simons (bottom). [15]. 


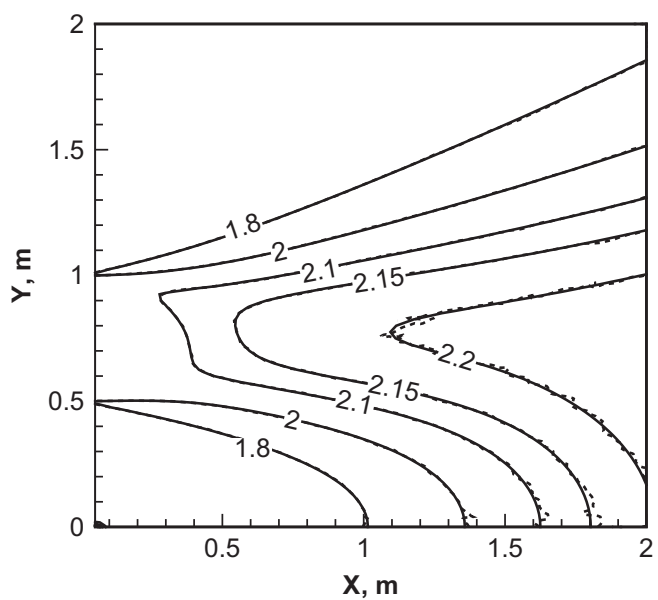

Fig. 6. Steady annular jet: normalized $U$-velocity contours, $S_{0}=2.0$, $R_{1}=1 \mathrm{~m}, R_{2}=0.5 \mathrm{~m}$.

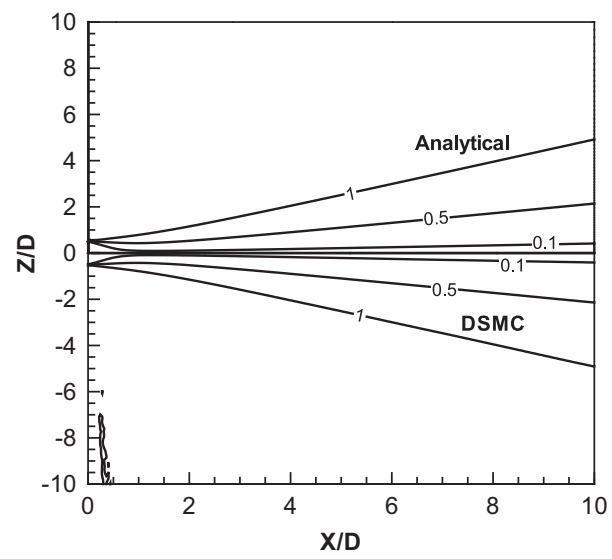

Fig. 7. Steady round jet: normalized $V$-velocity contours, $S_{0}=2.0$. [14].

$V$-velocity component must also be zero because no particles can reach that location without inter-molecular collisions. In the plume core around the centerline, the forward moving particles are dominating because of the high exit speed. Even though there are molecules with $V$-velocity component larger than zero, the small time period does not allows them to move upward too much.

Fig. 8 shows the steady jet pressure contours. The DSMC simulation and analytical results are almost identical, and the flow patterns are very similar to the density contours.

For the above contours we just discussed, the flowfield is steady, the scatters are low because we can achieve large sampling size by using both many time steps and also many particles, theoretically we can achieve an infinitely large sampling size so the flowfield has zero statistical scatters. However, for the unsteady flowfield evolution, the situations are different. The sampling size is limited by the number of particles we used during the simulation, and the time steps we used for sampling. The former is limited by the computer memory. As to the time steps, because it is very difficult to use ensemble simulations, we need to sample, starting earlier and ending later, than the time that we want to capture the flowfield. For example, if we want to capture the jet starting up flowfield at the very moment of $t / T_{*}=1.0$, where $T_{*}$ is the characteristic time period defined as $T_{*}=\left(R_{2}-R_{1}\right) / \sqrt{2 R T_{0}}$ for an annular exit in this paper, we may need to sample within $0.95<t / T_{*}<1.05$. The results for unsteady flowfield may have larger statistical scatters due to an insufficient sampling size, and we can only expect the analytical and DSMC simulation results with fair agreement in this paper.

The next pictures are about unsteady round or annular jet starting up or shutting down flows into a vacuum. Figs. 9-12 show the unsteady development of jet shutdown at the time $t / T_{*}=0.25$. Compared with the steady state, the DSMC simulation results have large statistical scatters. Because faster molecules leave the simulation domain quickly, the remaining particles have lower macroscopic velocities which result in relatively larger the scatters. By comparisons, the analytical results offer us zero statistical scatter results, which theoretically can only be obtained by DSMC with an infinitely large sampling size. All the flowfield patterns on the left close to the exit are mild or smooth, and no singularity points exist. These

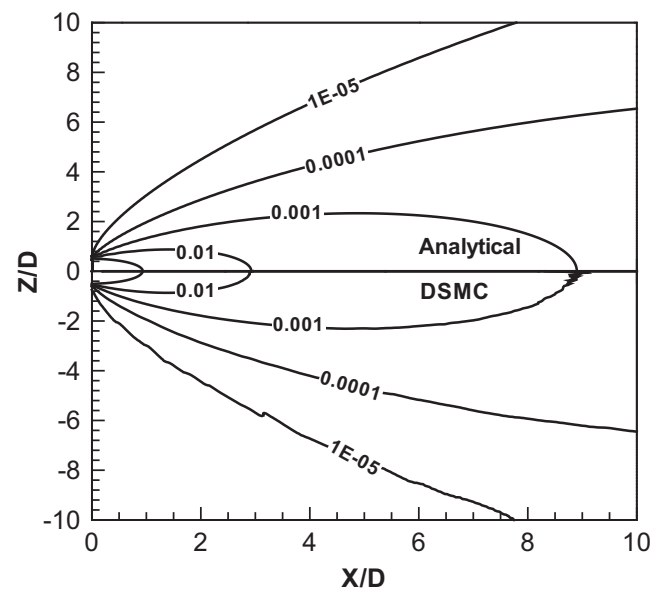

Fig. 8. Steady round jet: normalized pressure contours, $S_{0}=2.0$. [14].

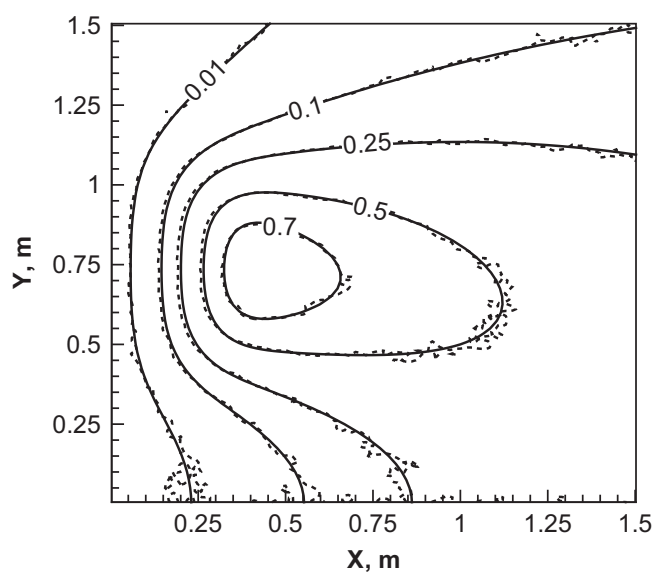

Fig. 9. Annular jet shutdown: $n(X, Y)$ contours, $t / T_{*}=0.25 ; S_{0}=2, R_{1}=$ $1.0 \mathrm{~m}, R_{2}=0.5 \mathrm{~m}$, dashed: DSMC, solid: analytical. 


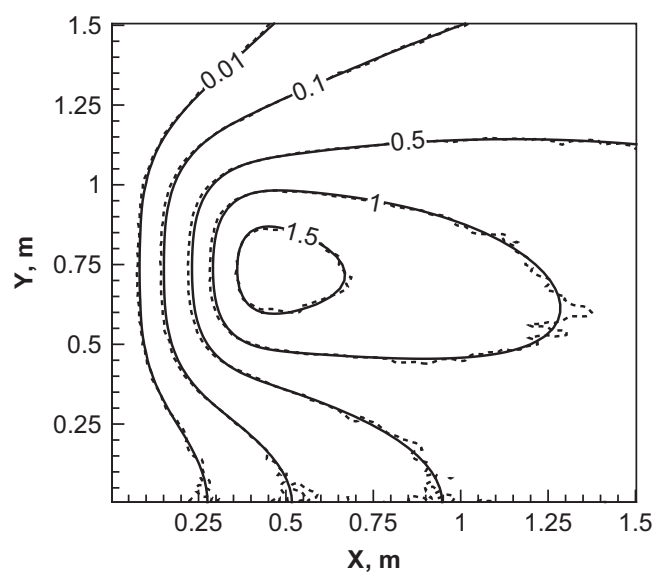

Fig. 10. Annular jet shutdown, $X$-momentum contours, $t / T_{*}=0.25 ; S_{0}=2$, $R_{1}=1.0 \mathrm{~m}, R_{2}=0.5 \mathrm{~m}$, dashed: DSMC, solid: analytical.

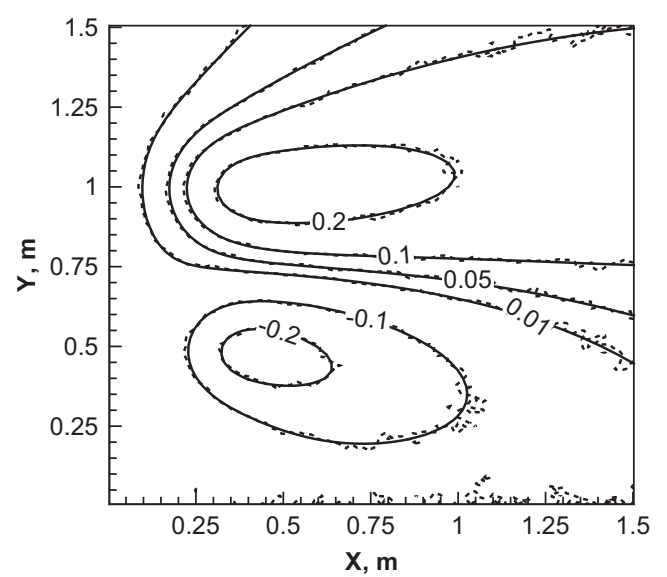

Fig. 11. Annular jet shutdown: $Y$-momentum contours, $t / T_{*}=0.25 ; S_{0}=2$, $R_{1}=1.0 \mathrm{~m}, R_{2}=0.5 \mathrm{~m}$, dashed: DSMC, solid: analytical.

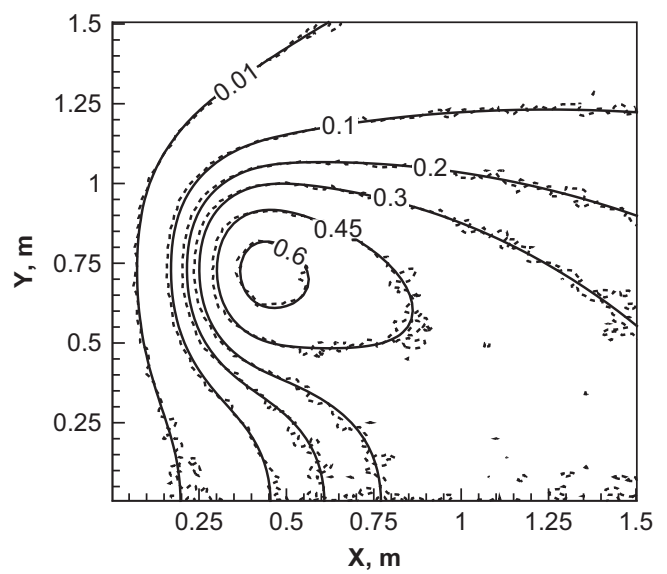

Fig. 12. Annular jet shutdown: pressure contours, $t / T_{*}=0.25 ; S_{0}=2$, $R_{1}=1.0 \mathrm{~m}, R_{2}=0.5 \mathrm{~m}$, dashed: DSMC, solid: analytical.

smoother flow patterns indicate that the flowfield contains more uniform speed particle after a shutdown process starts, in fact, only slow particles can stay in the flowfield

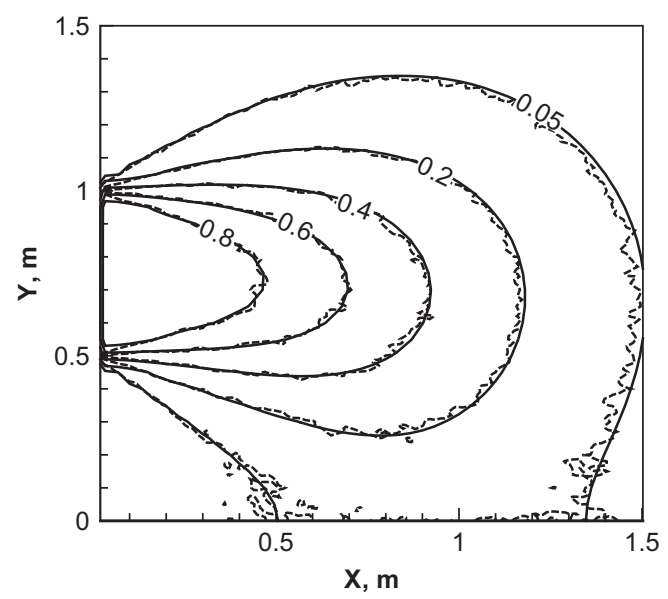

Fig. 13. Annular jet startup: contours of $n(X, Y), t / T_{*}=1.0, S_{0}=2$, $R_{1}=1.0 \mathrm{~m}, R_{2}=0.5 \mathrm{~m}$, dashed: DSMC, solid: analytical.

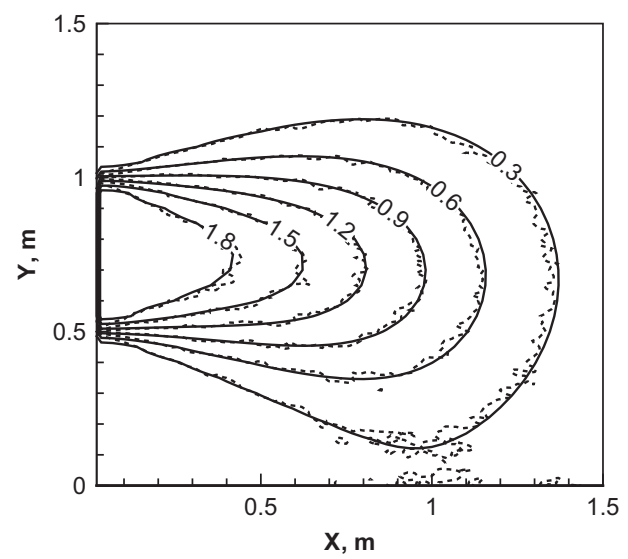

Fig. 14. Annular jet startup: $X$-momentum contours, $t / T_{*}=1.0, S_{0}=2$, $R_{1}=1.0 \mathrm{~m}, R_{2}=0.5 \mathrm{~m}$, dashed: DSMC, solid: analytical.

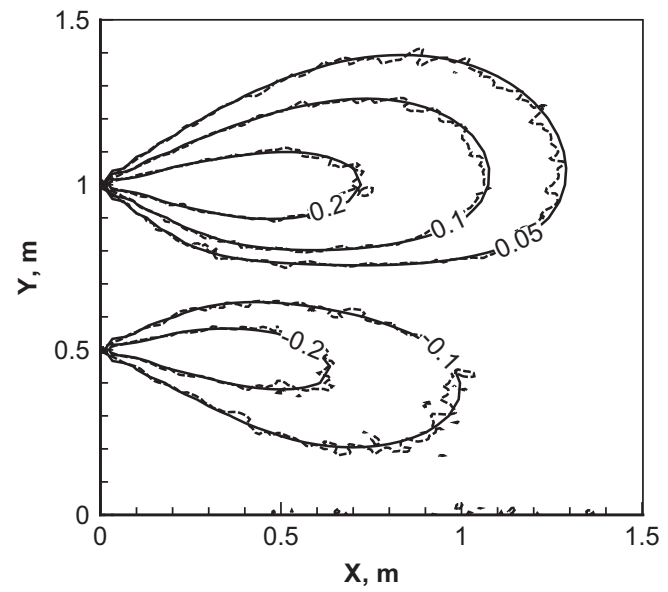

Fig. 15. Annular jet startup: $Y$-momentum contours, $t / T_{*}=1.0, S_{0}=2$, $R_{1}=1.0 \mathrm{~m}, R_{2}=0.5 \mathrm{~m}$, dashed: DSMC, solid: analytical. 


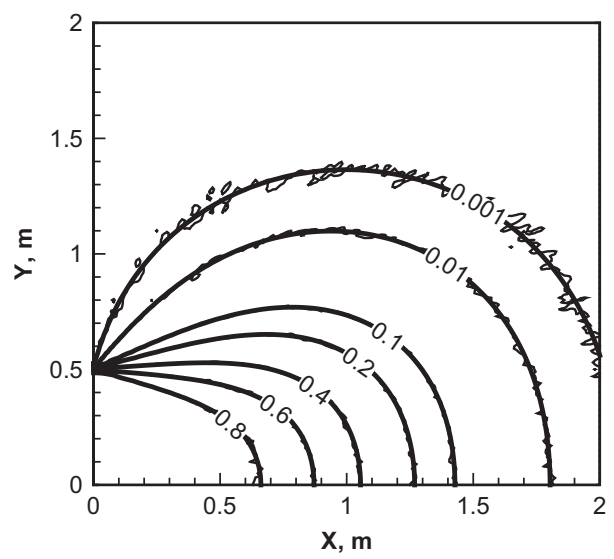

Fig. 16. Round jet startup: pressure contours, $t / T_{*}=1.0, S_{0}=2$, round exit radius $R=0.5 \mathrm{~m}$, dashed: DSMC, solid: analytical.

with a longer time. Figs. 13-16 show a startup process of density, $X$-, $Y$-momentum and pressure development at $t / T_{*}=1.0$. As time goes by, a jet expands into a vacuum, the analytical, DSMC simulation results are sufficiently close to validate the startup stage formulas.

\section{Summary}

In this study, a gaskinetic model for unsteady, high speed, free molecular gas flowing out of a circular/annular exit is investigated analytically and the results are validated by DSMC particle simulations. One velocity-position-time relation, Eq. (14), guided us to obtain the unsteady flowfield solutions. The time factor works as a limiter to determine the integration domain. This study yielded a full set of analytical solutions for the properties at an arbitrary flowfield point. These analytical and particle simulation results capture salient features of the entire flow field.

Because these analytical results include exit geometry, $R_{1}$ and $R_{2}$, time, and exit speed ration $S_{0}$, as such they are for the full flowfield and more comprehensive than many current plume models. For example, the widely used traditional cosine law/Boynton/Simons plume model is merely a point source, density only, stead plume model.

This study also revealed that there are very simple complementary relations among the density, momentum properties for the jet startup, steady, and shutdown process, although these results themselves are very complex. The solutions can find applications in micro-manufacturing, materials processing, and space engineering. They offer physical insights into the transitional gas flow regime (from collisionless to near continuum), less computer memory, zero statistical scatters, and a reduction in computation time.

\section{Acknowledgments}

We would like to acknowledge the financial support from NSF-DMS-0914706, New Mexico Space Grant, and NSF-CBET-0854411, and some helpful comments from Dr. M. Woronowicz.

\section{Appendix: Several used complex integrations}

$$
\begin{aligned}
& \int_{0}^{a} e^{-s^{2} / Q+2 s S_{0}} d s=\frac{\sqrt{\pi Q}}{2} e^{S_{0}^{2} Q} A, \\
& \int_{0}^{a} s e^{-s^{2} / Q+2 s S_{0}} d s=\frac{Q}{2}[1-B]+S_{0} \frac{\sqrt{\pi Q^{3}}}{2} e^{S_{0}^{2} Q} A, \\
& \int_{0}^{a} s^{2} e^{-s^{2} / Q+2 s S_{0}} d s=\frac{Q^{2} S_{0}}{2}-\left(\frac{Q a}{2}+\frac{Q^{2} S_{0}}{2}\right) B \\
& +\frac{\sqrt{\pi Q^{3}}}{4} e^{S_{0}^{2} Q}\left(1+2 S_{0}^{2} Q\right) A, \\
& \int_{0}^{a} s^{3} e^{-s^{2} / Q+2 s S_{0}} d s=\frac{Q^{2}}{2}\left(1+Q S_{0}^{2}\right) \\
& -\frac{Q}{2}\left(a^{2}+Q+Q S_{0} a+Q^{2} S_{0}^{2}\right) B \\
& +\left[3+2 S_{0}^{2} Q\right] \frac{\sqrt{\pi Q^{3}}}{4} e^{S_{0}^{2} Q} Q S_{0} A, \\
& +\frac{\sqrt{\pi Q^{3}}}{4} e^{S_{0}^{2} Q}\left(\frac{3}{2} Q+6 Q^{2} S_{0}^{2}+2 Q^{3} S_{0}^{4}\right) A . \\
& \int_{0}^{a} s^{4} e^{-s^{2} / Q+2 s S_{0}} d s=\frac{5 Q^{3} S_{0}}{4}+\frac{Q^{4} S_{0}^{3}}{2}-\frac{Q}{2} B \\
& \left(a^{3}+\frac{3}{2} Q a+\frac{5}{2} Q^{2} S_{0}+a^{2} Q S_{0}+Q^{2} S_{0}^{2} a+Q^{3} S_{0}^{3}\right)
\end{aligned}
$$

Here we define the following symbols to simplify the expressions:

$A=\operatorname{erf}\left(\frac{a}{\sqrt{Q}}-\sqrt{Q} S_{0}\right)+\operatorname{erf}\left(\sqrt{Q} S_{0}\right), \quad B=e^{-a^{2} / Q+2 a S_{0}}$.

\section{References}

[1] R. Campargue, Historical account and branching to rarefied gas dynamics of atomic and molecular beams, in: M. Capitelli (Ed.), 24th International Symposium on Rarefied Gas Dynamics, American Institute of Physics, New York, 2005.

[2] H. Pauly, Atom, Molecule, and CLuster Beams I, Springer, Berlin, Germany, 2010.

[3] R. Maev, V. Leshchynsky, Introduction to Low Pressure Gas Dynamic Spray, Wiley-Vch, Weinheim, Germany, 2008.

[4] C. Brook, M.L.R. Walker, Effect of anode temperature on hall effect thruster performance, J. Propul. Power 26 (September-October (5)) (2010) 1036-1044.

[5] M. Micci, K. Ketsdever, Micropropulsion for small spacecraft, Prog. Astronaut. Aeronaut. 187 (2000).

[6] H. Liepmann, Gaskinetics and gasdynamics of orifice flow, J. Fluid Mech. 10 (1961) 65-79.

[7] R. Narasimha, Orifice flow at high Knudsen number, J. Fluid Mech. 10 (1961) 371-384.

[8] R. Narasimha, Collisionless expansion of gases into vacuum, J. Fluid Mech. 12 (1962) 294-308.

[9] J. Brook, Density field of directed free-molecular flow from an annulus, J. Spacecr. 6 (1969) 755-757.

[10] M. Woronowicz, Initial molecular flow observations on bipolar planetary nebulae gaskinetics, in: AIAA Paper 2000-0599, 38th Aerospace Sciences Meeting and Exhibit, 2000, Reno, Nevada.

[11] F.P. Boynton, Exhaust plumes from nozzles with wall boundary layers, AIAA J. 1 (1967) 1703.

[12] G.A. Simons, Effects of nozzle boundary layers on rocket exhaust plumes, AIAA J. 10 (11) (1972) 1534-1535. 
[13] G. Dettleef, Plume flow and plume impingement in space technology, Prog. Aerosp. Sci. 28 (1) (1991) 1-71.

[14] C. Cai, I.D. Boyd, Collisionless gas flow expanding into vacuum, J. Spacecr. Rockets 44 (6) (2007) 1326-1330, http://dx.doi.org/ 10.2514/1.3217173.

[15] C. Cai, L. Wang, Numerical validations for a set of collisionless rocket plume solutions, J. Spacecr. Rockets 49 (1) (2012) 59-68, http://dx. doi.org/10.2514/1.A32046.

[16] G.A. Bird, Molecular Gas Dynamics and the Direct Simulation of Gas Flows, Oxford University Press, New York, 1994.
[17] C. Cai, X. Huang, High speed rarefied round jets and jet impingement, AIAA J. 50 (December (12)) (2012) 2908-2918.

[18] E. Bender, Free molecular expansion of initially moving gases into vacuum, Quart. J. Mech. Appl. Math. 19 (4) (1966) 497-509.

[19] H. Liu, C. Cai, C. Zou, An object-oriented implementation of a DSMC simulation package, Comput. Fluids 57 (1) (2012) 65-75, http://dx. doi.org/10.1016/j.compfluid.2011.12.007. 